\title{
A Survey of Wireless Communication Technologies \& Their Performance for High Speed Railways
}

\author{
Subharthi Banerjee, Michael Hempel, Hamid Sharif \\ Department of Electrical \& Computer Engineering, University of Nebraska-Lincoln, Omaha, NE, USA \\ Email: sbanerjee15@huskers.unl.edu,mhempel@unl.edu, hsharif@unl.edu
}

Received 30 September 2015; accepted 5 January 2016; published 8 January 2016

Copyright (C) 2016 by authors and Scientific Research Publishing Inc.

This work is licensed under the Creative Commons Attribution International License (CC BY).

http://creativecommons.org/licenses/by/4.0/

(c) (i) Open Access

\section{Abstract}

High Speed Railway (HSR) provides its customers not only safety, security, comfort and on-time commuting, but also a fast transportation alternative to air travel or regular passenger rail services. Providing these benefits would not be possible without the tremendous growth and prevalence of wireless communication technologies. Due to advances in wireless communication systems, both trains and passengers are connected through high speed wireless networks to the Internet, data centers and railroad control centers. Railroad communities, academia, related industries and standards bodies, even the European Space Agency, are involved in advancing developments of HSR for highly connected train communication systems. The goal of these efforts is to provide the capabilities for uninterrupted high-speed fault-tolerant communication networks for all possible geographic, structural and weather conditions. This survey provides an overview of the current state-of-the-art and future trends for wireless technologies aiming to realize the concept of HSR communication services. Our goal is to highlight the challenges for these technologies, including GSM-R, Wi-Fi, WIMAX, LTE-R, RoF, LCX \& Cognitive Radio, the offered solutions, their performance, and other related issues. Currently, providing HSR services is the goal of many countries across the globe. Europe, Japan \& Taiwan, China, as well as North \& South America have increased their efforts to advance HSR technologies to monitor and control not only the operations but also to deliver extensive broadband solutions to passengers. This survey determined a trend of the industry to transition control plane operations towards narrowband frequencies, i.e. LTE400/700, and to utilize concurrently other technologies for broadband access for passengers such that services of both user and train control systems are supported. With traditional technologies, a tradeoff was required and often favored train control services over passenger amenities. However, with the advances in communication systems, such as LTE- $R$ and cognitive radios, it is becoming possible for system designers to offer rich services to passengers while also providing support for enhanced train control operations such as Positive Train Control. 
Keywords

High Speed Railways, HSR, Broadband Access, High Mobility, Cognitive Radio

\section{Introduction}

High Speed Railways have been deployed for many years. At present, HSR is competing with domestic and international flight operators for passengers. Efforts and developments in this area, however, cannot only focus on fast transportation as an attractor, but also ensuring safety and security of its passengers, as well as convenience features such as seamless Internet service and rich entertainment offerings.

Enabled by advances in wireless communication technologies, these services are the key to successful HSR operations. But HSR maintains a non-stationary channel model with high-speed predetermined mobility, where the mobility path is dictated by the installed track network. Consequently, a wired approach is not feasible to connect a train with its infrastructure network. Physical guided media, like wires, are not suitable for HSR communication networks, as a result of high voltage lines, physical vibrations \& harsh temperature environments. Thus, ground to train communication employs or proposes various wireless standards. To ensure successful integration, these wireless standards have to be reliable and robust. Applications such as on-board video surveillance, train control operations, signaling, diagnostics \& monitoring are becoming increasingly bandwidth intensive and complex. Data prioritization is therefore also of importance. Train control operations require a lower data rate than, for example, video streaming services, but are of significantly higher priority.

HSR in Europe, Asia and North America is different from each other. In North America, freight train operators are the major factor for rail services. Freight trains are slow-moving and require network services primarily for train operations and control. In Europe, train operators started making Internet access available to passengers in 2005 [1]. But in the US, although there is a densely connected railroad network, the majority of rail operators are freight carriers. A notable exception is the Northeast corridor, where Amtrak is a dominant presence in providing high speed rail services between the major cities along the East Coast. The passenger count in 2014 alone was 12 million. However, its broadband access service for passengers is less developed than its European counterparts. To remedy this situation, significant research efforts are underway to provide both freight and passenger railways high speed wireless network capabilities. Cognitive Radio (CR) can significantly improve the network performance, while systems for Positive Train Control (PTC) can provide overall train control \& safety services.

A train is considered to be a high speed train if it is capable of achieving a speed of more than $200 \mathrm{~km} / \mathrm{hr}$. At that speed it is a challenging task to maintain a stable communication network between Base Station (BS) and train due to propagation and channel effects, cell handovers, and more. High speed train wireless networks face challenges not only due to high node mobility but also from track-side obstacles such as foliage or buildings, from railcar construction, terrain topology and others. Most railway operators thus favor network heterogeneity to offer seamless connectivity to their passengers. Sometimes, this acts as a "gap-filler", as described in Section 3.6, at the cost of increased infrastructure expenses. Table 1 shows HSR operators selecting different technologies for their trains.

In this survey, we will review wireless communication technologies researched or deployed in the high speed rail domain. In Chapter 2, we are exploring communication needs in HSR, followed by a review of the historical development and potential future trends in Chapter 3. Chapter 4 states the challenges that wireless communication networks face in HSR scenarios. Finally, Chapter 5 provides our conclusion.

\section{Communication Requirements in High Speed Railways}

Broadband access and reliable communication channels have become an imperative part of HSR. Train operations, control, diagnostics, and telematics highly depend on communications. Trains are considered "connected" when they are in communication with train control and dispatch centers, signaling zones, level crossings, stations and even provide communication among railcars \& trackside equipment. This basic real-time communication does not require a significant amount of bandwidth or data rate. But robust and reliable communication is paramount for train operations. Advanced communication systems often are employed to mitigate the potential for human errors. 
Table 1. Applied wireless technologies in high speed trains.

\begin{tabular}{|c|c|c|c|c|}
\hline High Speed Trains & Country & Key Cities \& Routes & $\begin{array}{l}\text { Avg. Speed (km/hr.)/Max. } \\
\text { Speed (km/hr.) }\end{array}$ & $\begin{array}{c}\text { Key Wireless } \\
\text { Technologies Used }\end{array}$ \\
\hline TGV-POS (SNCF) & France & $\begin{array}{c}\text { Basel, Zurich, } \\
\text { Vallorbe, Lausanne }\end{array}$ & $320 / 574.8$ & ETCS-2/Satellite \\
\hline $\begin{array}{c}\text { CRH380A \& AL } \\
\text { (China Railway Corporation) }\end{array}$ & China & $\begin{array}{l}\text { Shanghai-Hangzhou } \\
\text { Shanghai-Nanjing }\end{array}$ & $300 / 487.3$ & GSM-R \\
\hline AVE Class 103 (RENFE) & Spain & Madrid, Taragona & $310 / 403.7$ & GSM-R (ETCS-2) \\
\hline $\begin{array}{l}\text { ICE } 3 \text { class 403, } 406 \text { (Deutsche } \\
\text { Bahn, Nederlandse Spoorwegen) }\end{array}$ & Germany & Frankfurt, Paris & $330 / 368$ & $\begin{array}{l}\text { GSM-R, Radiating cable } \\
\text { (LCX), Wi-Fi }\end{array}$ \\
\hline ETR 500 (Trenitalia) & Italy & $\begin{array}{l}\text { Rome, Naples, } \\
\text { Florence, Bologna }\end{array}$ & $300 / 362$ & $\begin{array}{l}\text { Radiating cables, Wi-Fi, } \\
\text { GSM-R }\end{array}$ \\
\hline KTX-I & South Korea & Seoul, Busan & 330 & $\begin{array}{l}\text { ATC (Automatic Train } \\
\text { Control), Wi-Fi }\end{array}$ \\
\hline Eurostar (GNER, SNCF) & $\begin{array}{l}\text { France, Spain, } \\
\text { England }\end{array}$ & Brussels, Lille, London. & $300 / 334.7$ & Wi-Fi, WIMAX, ETCS-2 \\
\hline $\begin{array}{c}\text { N700 Shinkansen } \\
\text { (JR West, JR Central, JR Kyushu) }\end{array}$ & Japan & $\begin{array}{l}\text { Tokyo, Osaka, } \\
\text { Tokaido-Sanyo }\end{array}$ & $275 / 332$ & LCX \\
\hline THSR 700T & Taiwan, China & Taoyuan, Hsinchu, Taichung & 300 & RoF, WIMAX \\
\hline Acela (Amtrak) & USA & $\begin{array}{c}\text { Washington, Baltimore, New } \\
\text { York }\end{array}$ & $240 / 265$ & Wi-Fi, GSM-R \\
\hline Pendolino & Finland & Helsinki, Tampere & 250 & Flash-OFDM, WIMAX \\
\hline
\end{tabular}

\section{User Plane, Control Plane \& Architecture of the Network}

Broadband access from trains can be separated as two planes according to their traffic integrity requirements. The traffic can be train-focused (control plane) or passenger-focused (user plane). Train-related traffic provides services for train telematics, control, signaling, and diagnostics data. Passenger traffic contains VoIP, Internet browsing, video streaming, games and other infotainment data. Compared with train traffic, passenger traffic has vastly higher bandwidth requirements. However, the train-focused traffic is of higher priority, because protecting the integrity and delivery of this traffic is more important than high performance. Thus there exists the need for a tradeoff between these two planes when realized in communication systems.

The problem is addressed by several researchers. Li Yan et al., for example, suggested a User-Plane/ControlPlane decoupled architecture to better manage the wireless network [2]. Li Yan argued that higher frequency and wide band spectrum allocation along the user-plane separation will increase the capacity of the network while also maintaining reliability. Li Yan et al. also focused on control and data signaling plane separation [3]. The goal of their work is to keep the C-Plane within the existing narrow-band wireless network and have the U-plane take advantage of wide-band networks with better handover characteristics. Handover occurs due to the train's movement from one cell to another. And for high speed trains handover happens frequently. An efficient algorithm is needed that optimizes the number of handovers in high speed mobility. The authors show that with their approach the performance in the network was greatly enhanced. This was achieved by basing this architecture on LTE-R and a U-Plane/C-Plane decoupling. Legacy network systems used to couple both planes together, which resulted in traffic congestion, especially in the absence of optimized network management functionality. LTE-R attempts to remedy this situation. With the help of System Architecture Evolution (SAE) it separates the two traffic planes logically. Mobility Management Entity (MME) and gateways can manage each of the logically separated planes. However, the accumulated traffic still lacks physical separation [2].

The legacy systems are operated through macro cells. Macro cells provide reliability in coverage and better connectivity in lower frequency bands. These are the characteristics leveraged for control plane traffic. On the other hand, the targeted frequency band of LTE is moving towards higher frequencies. These higher frequency bands utilize small cells (Femto \& Pico cells), which are more susceptible to propagation loss than macro cells and thus cannot cover large distances, and are infeasible for the control plane traffic but provide very high capacity for user plane traffic. That's why small cells are called "phantom cells". The authors therefore propose 
handling the real time control plane traffic over macro cells and resource intensive user plane traffic over small cells.

The communication network architecture for high speed rail resembles a tree topology, as shown in Figure 1, where service providers are situated at the top of the tree, representing the core network facilities. Situated below is the aggregation network and finally the access network. The access network is comprised of the trackside infrastructure for Train-to-Ground communication [1]. This access network is the key differentiator between different service technologies, and the focus of the next chapter.

\section{Evolution \& Trends of Wireless Communication in the HSR Access Network}

Train-to-ground communication has been an important focus in the research around HSR due to its high speed and the complications for wireless communication systems resulting from it. Traditional communication methods could not support high-speed rail deployments. Due to high train speeds, special track systems and installations are needed, such as maglev and viaducts. With this drastic change in infrastructure, a resulting change in communication infrastructure was necessary, in part because of inexperience in network and deployment design for these systems, and in part because the technologies did not adequately support these high train speeds. The European Train Control System (ETCS) was the first to study this problem in detail and endeavored to resolve it by having a standard digital communication method for all trains in Europe. At that time, 14 different analog train-to-ground communication systems existed in Europe and were consequently all replaced by ETCS and GSM-R.

There now exists a variety of standards for train control communication. Such systems include Communication Based Train Control (CBTC), Advanced Train Control System (ATCS), Command, Control and Communication

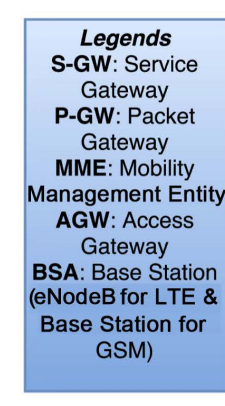

- Control data link for LTE

_ User data link for LTE GSM Link Direct satellite link

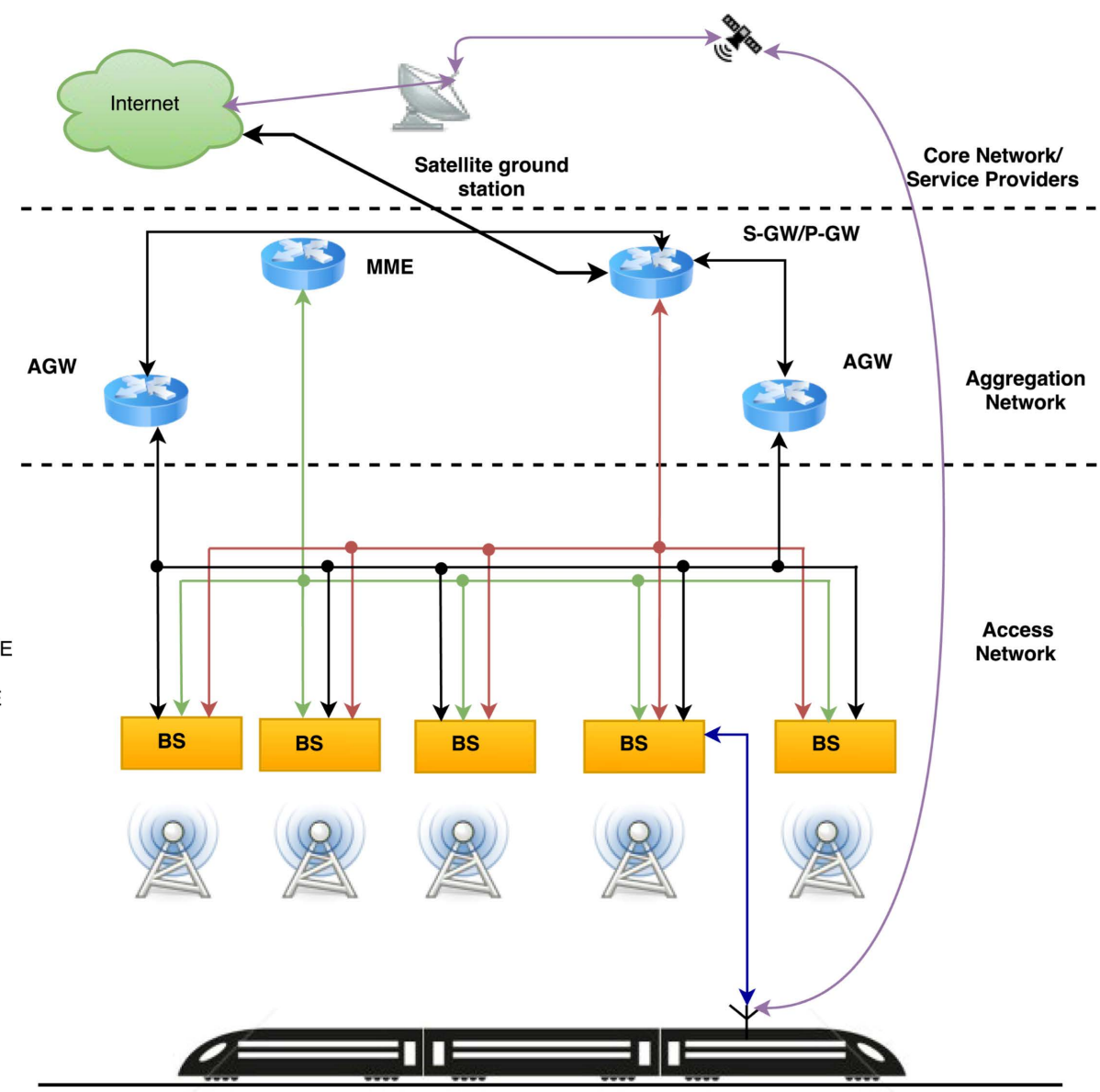

Figure 1. GSM-R and LTE-R architecture. 
Systems (CCCS), Incremental Train Control System (ITCS), Positive Train Control (PTC), as well as the aforementioned ETCS, all of which aim to make railway operations safer for its passengers [4]. ETCS defines transmission characteristics for the network [5]-[7] with the following parameters:

- Received signal power;

- End to end delay;

- Data rate;

- Probability of loss of connection;

- Maximum break during handover;

- Connection establishment delay or call set up time;

- Call establishment failure probability.

New technologies based on these standards have been defined in recent years. In the US, several new high speed rail deployments are planned \& deployed, such as the California High Speed Railways (CHSR) or Virginia High Speed Railways. This provides opportunities for novel and unique strategies and requirements for each deployment. They encompass significant research and development to overcome any potential challenges, which include wireless environment effects, installation obstacles of new infrastructure, new rail car and track designs, communication standardization, and ensuring safe, profitable, and timely operation.

\subsection{GSM-R}

GSM-R (GSM for railways) was created as an extension of GSM, with a focus on rail operations and to provide a standardized digital communications protocol that will be interoperable and cost-efficient. The aim was to replace analog systems traditionally used in the railroad environment with modern wireless all-digital systems. But the network had to follow some basic requirements for train communications, such as controller-driven communications, automatic train control, shunting and telemetry capabilities, emergency remote broadcast, local area communications, passenger services, location dependent addressing, and data-only radios.

GSM-R supports both voice \& data. Before the introduction of GSM-R, there was no standard to implement both voice and data in a digital channel. Since its introduction, railway operators from other continents have adopted GSM-R, primarily due to the lack of alternatives. Consequently, GSM-R rapidly grew in market share and importance. The first high speed rail service to support GSM-R was Germany's ICE from Saarbrücken to Paris, France. Subsequently, China, India, North Africa, and the US adopted GSM-R, at least in part, for their own deployments.

GSM-R has a multilevel preemption \& precedence model with a circuit switched Time Division Multiplexing (TDM) network. This allows the network to prioritize control plane data over user plane data. It is a narrowband technology defined to operate in the $800-900 \mathrm{MHz}$ band. The system permits the network to have better handover in macro cells, having base stations spaced approximately 6 - $10 \mathrm{~km}$ apart. It supports a maximum velocity of $500 \mathrm{~km} / \mathrm{h}$ and enables inter-country coverage due to its standardized frequency band.

Despite this overwhelming growth and maturity, GSM-R is approaching obsolescence. The International Union of Railways (UIC) is envisioning 4G technology to completely replace GSM-R by 2020. GSM and its related technologies are based on $2 \mathrm{G}$, and therefore cannot support the increasing bandwidth demand of modern Internet services and applications. Sniady et al. [6] looked into how GSM-R will be infeasible in the near future and found the primary reason to be the lack of support for the large expected client density, such as in train stations.

\subsection{Wireless LAN}

It has been 25 years since the introduction of IEEE 802.11, more commonly known as Wi-Fi. For in-train broadband access this represents the most widely used network technology. Wi-Fi works over the license-free ISM 2.4 GHz or 5.8 GHz frequency bands. It supports very large bandwidth, and provides high per-user capacity. In-train Wi-Fi has a very low packet loss rate [8] [9]. Combined with the ubiquitous presence of Wi-Fienabled devices including laptops and smart phones this provides an ideal solution for train operators. IEEE 802.11 is actually a family of protocols and now includes standards such as $802.11 \mathrm{a} / \mathrm{b} / \mathrm{g} / \mathrm{n} / \mathrm{ac}$. Terrestrial communication using Wi-Fi from high-speed trains is challenging due to its smaller cells. The range Wi-Fi covers is also very small (typically 250 - $450 \mathrm{~m}$ ). High handover frequency and the lack of a common approach to implement handovers using Wi-Fi make interoperability challenging. Finally, the Capital Expenditure (CAPEX) to deploy Wi-Fi over an entire track network is very high. 
However, IEEE 802.11 has accelerated the development of related protocols. Based on their physical \& MAC layer designs, these protocols are evolving into standards supporting vehicular networks. Examples include Bluetooth, WIMAX, WIMAX-2, Wireless Access Vehicular Environment (WAVE, comprised of IEEE 802.11p and IEEE 1609), ZIGBEE, and Dedicated Short Range Communications (DSRC). All of these are employed to provide safety, control, Internet access, health monitoring or diagnostics for the high speed vehicular environment. All these standards also support IP, which gives them an edge over GSM-R. DSRC\& WAVE are the most suitable for deployments in HSR. Salaberria et al. [10] advocated a unique concept of inter-railcar connection with Bluetooth. The research is a product of findings in industrial applications such as the Safe Driver Machine Interface (SAFEDMI) and On Board Wireless Video Surveillance (BOSS). Before downloading train diagnostics data and updating software in-train systems through wireless links, SAFEDMI tests the wireless links thoroughly. And BOSS is a proposed research solution in trains for CCTV surveillance utilizing IEEE 802.16.In the proposed architecture, the middleware consists of a Train Communication Manager (TCM) and a Ground Communication Manager (GCM) to distribute the active load of train-to-ground communication. The system includes a Bandwidth Management Service (BMS) to notify GCM of available bandwidth. It can be deployed using existing cellular and non-cellular technologies for robust operation.

Figure 2 illustrates an overall performance comparison of mentioned wireless technologies against high speed, before the survey analyze them further. Table 2 similarly shows a comparison of the maximum achievable throughput for these different technologies.

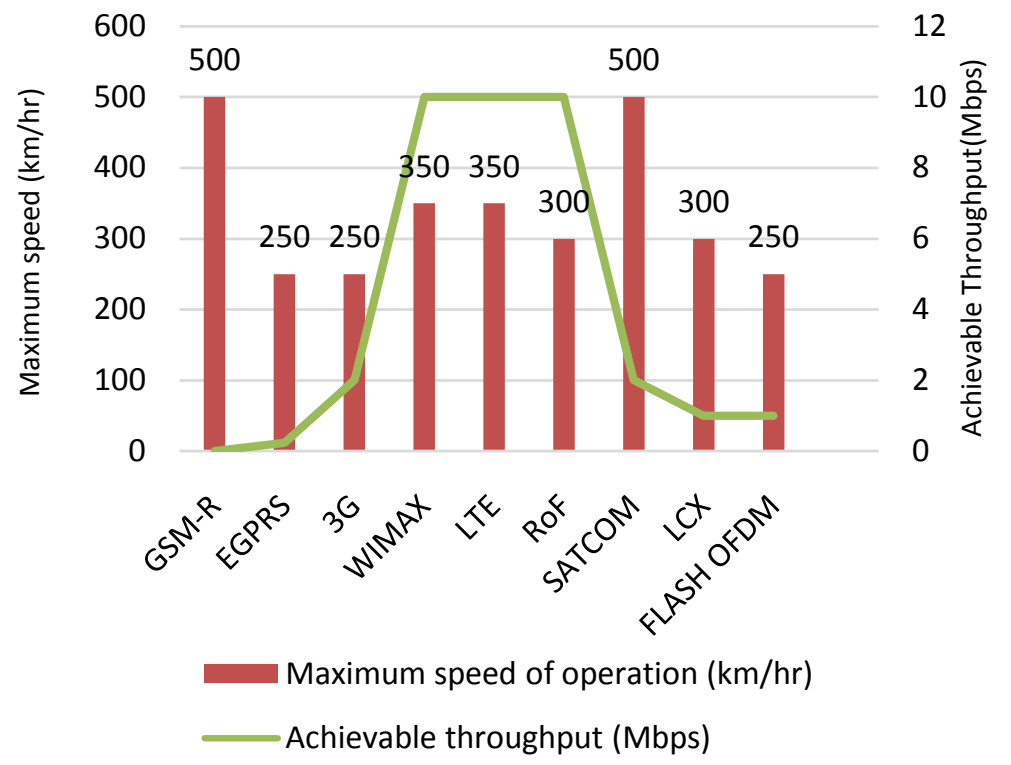

Figure 2. Performance of wireless technologies.

Table 2. Supported maximum throughput for various train communications technologies.

\begin{tabular}{cc}
\hline Technology & Maximum supported throughput \\
\hline GSM-R & $5 \mathrm{kbps}$ \\
EGPRS & $1 \mathrm{Mbps}$ \\
3G & $14.4 \mathrm{Mbps}$ \\
WiMAX & $>100 \mathrm{Mbps}$ \\
LTE & $>300 \mathrm{Mbps}$ \\
RoF & $>1 \mathrm{Gbps}$ \\
SATCOM & $>1 \mathrm{Gbps}$ \\
LCX & $>42 \mathrm{Mbps}$ \\
FLASH OFDM & $>5.2 \mathrm{Mbps}$ \\
\hline
\end{tabular}




\subsection{G \& LTE: Development of LTE-R}

As described in Section 3.1, GSM-R/2G is transitioning to $4 \mathrm{G}$ network deployments. WIMAX, and now WIMAX-2, have been an integral part of the transition to $4 \mathrm{G}$ networks. As a backhaul network it provides the infrastructure support for major high speed rail networks in Asia and Europe. Nomad Digital is one of the market leaders in providing broadband access to its major clients, including Acela, Amtrak, Eurostar, TGV, Canada's Via Rail, etc., all of which utilized WIMAX networks extensively. The release of WIMAX-2 elevated WIMAX into the group of $4 \mathrm{G}$ technologies. Over the years a lot of studies have been conducted on WIMAX and its applicability for railroad scenarios, such as our group's test bed in Crete, NE, developed and operated in collaboration with BNSF and support from the Federal Railroad Administration [11]. More recently, ITRI has been successful in deploying WIMAX for Taiwan High Speed Railways (THSR). The network runs successfully in a 300 $\mathrm{km} / \mathrm{h}$ high speed rail environment with high data rates.

Another 4G technology is 3GPP's Long Term Evolution, also known as LTE. LTE-supported architectures\& devices are now dominating in the commercial market. LTE can work as a backbone core network or directly work as access network for any vehicular communication technology. The dynamic behavior of LTE has compelled both rail operators and other related industries to utilize it in their systems. LTE has several advantages over WIMAX. As it can operate over various different bandwidths, existing GSM-R/TETRA networks can easily be upgraded to LTE-R, the railroad-specific extension of LTE. Recently, Alcatel-Lucent \& Cassidian [8] mentioned their interest in the LTE400 band. This is indicative of the current trend to move towards lower frequencies for critical control plane applications. This makes it possible to extend radio coverage over larger distances with lower CAPEX \& Operational Expenditure (OPEX).

LTE-R has clear advantages over GSM-R, as shown in [6] [7], such as better control over network resources, packet differentiation based Quality of Service (QoS) mechanism, reduced packet delay, high throughput and IP support.

Advanced modulation \& multiplexing made LTE the better choice compared to GSM and other technologies. However, in order to accommodate the LTE-R architecture as part of the ETCS standard, ETCS needs to redefine their specifications. Sniady et al. have reported that the Danish signaling program has changed some of the ETCS specifications according to the packet switched network parametric specifications. They have added several parameters so that they can evaluate LTE performance in rail, including transfer delay \& transmission delay, data integrity with packet loss probability, duplicity, out-of-sequence packet delivery, processing delay, queuing delay, as well as the activation delay for Packet Data Protocol (PDP).

When the system was tested with LTE-R at the $54 \mathrm{~km}$ Snoghøj-Odense track, it performed within ETCS specifications. Evolved Packet Core (EPC) and UMTS Terrestrial Radio Access Network (E-UTRAN) comprise the core of the LTE network. EPC is based on a multi-access network architecture with 3GPP and non-3GPP technologies like WLAN. With Self-Optimizing Network (SON) functions for configuration, optimization and carrier aggregation, LTE also can achieve better performance in rail networks. LTE has the compatibility to carry out both U-plane/C-plane or "vital \& non-vital" operations.LTE networks support various different frequencies and different bandwidths. The capacity of the network is almost 300 Mbps. Orthogonal Frequency Division Multiplexing (OFDM) is used for downlink, while Single Carrier Frequency Division Multiplexing (SC-FDMA) is used for uplink to support high capacity. In terms of capacity, only Radio over Fiber, described in the next chapter, can compete with LTE.

\subsection{Radio over Fiber (RoF)}

RoF is a recent advance in high capacity broadband access for railways. The aggregate capacity can reach up to 1 Gbps using RoF. The "Pico cells" introduced in RoF can enhance bandwidth utilization for the large number of users as with statistical multiplexing [12]. Pleros et al. [13] have explained their research on $60 \mathrm{GHz}$ RoF, which operates over $60 \mathrm{GHz}$ frequency and relies on millimeter-wave technology. They described phenomena with mobile users, i.e. having an indoor scenario with additional attenuation, sharp bends with sudden loss of connectivity from the Remote Antenna Unit (RAU), etc. The $60 \mathrm{GHz}$ signal restricts the pico-cell radius to about $10 \mathrm{~m}$, which can cause handover issues for even slow moving users. The noted solution for this sort of problem is Dynamic Capacity Allocation (DCA), where the "physical layer takes a wavelength routing approach". Static Extended Cell (SEC) \& Virtual Cellular Zone (VCZ) provide seamless connectivity in indoor and outdoor situations. But the most important concept is the "moving cell". SEC \& VCZ both increase cell overlapping area em- 
ploying static, adjacent cells and have a better Call Dropping Rate (CDR).The concept describes an adaptable cellular technique, synchronizing with the moving user's speed and direction. The suggested "Moving Extended Cell (MEC)" concept, on the other hand, employs "user centric virtual groups of adjacent cells" for transmitting the same data content from multiple physical cells. MEC uses a switch structure to reconstruct the "virtual multi-cell area". The restructuring happens based on the user's mobility pattern, so that a "virtual antenna group" moves with the users. Using this MEC concept, the authors could simulate a capacity of over 100Mbps in 60 $\mathrm{GHz}$ with zero packet loss. An increasing number of vendors are utilizing RoF technology for providing high speed railway solutions. Greve et al. proposed a seamless connectivity for "Fast Moving Users (FAMOUS)" with RoF [14]. The architecture establishes a ring network with a control station as a base and distributed antenna systems as other nodes. Those distributed antenna systems or Remote Antenna Units (RAU) are connected over an optical fiber link. The handoff problems are mitigated due to the "moving cell". Lanoo et al. have argued [12] that the solution has a distinct advantage over the classical networks and stated that GSM-R depends on base stations and UMTS on node-B, whereas for RoFthe control station and RAU can distribute the load of BS and node-B among its nodes. RAU can work passively with dedicated frequency allocation and wave division multiplexing.

Han et al. recommended a novel solution for high speed railways based on RoF, which is RADio-Over-fiber as AnTenna Extender (RADIATE) [15]. They stated that 78\% of regular high-speed train commuters are interested in Wi-Fi enabled trains and 72\% would prefer HSR over flights if Wi-Fi is available on-board. Han et al. have studied this situation and designed RADIATE to work in train systems with existing cellular backhaul. The authors claim that during an LTE Round Trip Time (RTT) of 70 - $140 \mathrm{~ms}$, a high speed train can move up to 10 $m$. RADIATE is designed to minimize RTT, with its dense on-roof antenna system.

\subsection{Leaky Coaxial Cable (LCX)}

LCX is widely used in Japan. Tokyo's largest Shinkansen network, Tokaido-Shinkansen provides Internet access to passengers through LCX with IPV4 support [16]. According to the information provided in [16], there are around 59 LCX transceivers between Tokyo \& Hakata station covering 1069 km in Tokaido \& Sanyo Shinkansen. Leaky cable or radiating cables in Japan deployed for trains provides an Ethernet extension that is called Mobile Ethernet [1]. Mobile Ethernet allows the routers and switches to learn and adapt to the communication, which reduces unnecessary broadcasts. The cables follow the maglev tracks and radiate RF signals as the access network.

According to [16], the Mobile Router (MR) acts as access point inside the train. Shinkansen LCX access points can provide a capacity averaging $0.7 \mathrm{Mbps}$ in up-link and down-link with Network Mobility Extensions for Mobile-IPV4 (NEMO-v4). The goal, however, is to achieve 1 Gbps capacity employing IPV6 with LCX and Infrared (IR). IR has a bandwidth capacity of 1 Gbps and a range of $300 \mathrm{~m}$. Due to this small range, IR is prone to more handover failures than LCX cables. Terada et al. have proposed to use in-train Infrared Communication Devices (IR-CD) with Network mobility Basic Support protocol (NEMO BS), which supervises fast handover in the system.

Guan et al. argue that a network has the highest efficiency when the signal spreads evenly in the railcars [17]. They endorse the idea of having LCX all over the train. Because the loss in indoor condition is lower than the free space loss. For an LCX cable, each leakage that radiates electromagnetic waves can act like an individual antenna. The leakage is formed due to slots in the RF coaxial cable. For an operating frequency of $2.4 \mathrm{GHz}$, these grooves are spaced less than $p=58.9 \mathrm{~mm}$ apart. And can be determined as found in [17],

$$
-\frac{c}{1+\sqrt{\varepsilon_{r}}} \cdot \frac{m}{p}<f<-\frac{c}{\sqrt{\left(\varepsilon_{r}-1\right)}} \cdot \frac{m}{p}
$$

where $\varepsilon_{r}$ is the equivalent dielectric constant; $c$ the speed of light; and $f$ is the operating frequency. And surface waves can only produce radiation when $m \leq-1$.

\subsection{Satellite Communication}

SATellite Communication (SATCOM) systems are used heavily in sparsely populated areas due to the lack of terrestrial communication infrastructure. This method of communication is also irreplaceable for airborne traffic. But for fast moving users on the ground, the advantages and disadvantages for SATCOM both must be consi- 
dered. Communication satellites provide a vast coverage area, which can enable Internet access in combination with aggregation networks. Geostationary Earth Orbit (GEO) satellites are most commonly used for train communication. Despite having a long propagation delay of more than 500 - $600 \mathrm{~ms}$, and significant cost of implementation, GEOs are employed in railways network. SATCOM signals have a limited selection of frequency bands and cannot cover non-line-of-sight zones like hilly terrains, bad weather affected zones, tunnels and even densely populated urban areas. In such scenarios train operators deploy "gap fillers" to avoid interruptions [18]. There are two kind of "gap fillers" widely used in European HSR scenario:

Satellite Repeaters: Installed antenna can be used to recover signals in non-line of site zones.

Vertical Handover: Another technology should be used in place of SATCOM for seamless connectivity.

Satellite communications also face significant burst errors due to obstacles and mobility. The larger the obstacle, the larger the impact on the signal [18]. For cellular networks, a high velocity increases propagation channel errors in cellular networks. But for SATCOM, a lower the speed results in larger burst error rates.

At the time of French National Railway Company's (SNCF) SATCOM testing, Icomera \& Pointshot had hybrid satellite systems whereas 2-way satellite communication systems were marketed by 21Net, Telespazio, Viasat/Rays at and Opensat/Accorde [18]. During their testing SNCF determined that hybrid cellular SATCOM is not suitable for deployments in HSR. But using two-way SATCOM broadband access could be established concurrently in places where cellular coverage is inadequate. SNCF therefore chose the two-way SATCOM to be their train-to-ground communication network, which interfaces with Wi-Fi access points as "gap-filler".

Intelligent Railways via Integrated Satellite Services (IRISS) is a project of the European Space Agency (ESA) Basic objectives of IRISS are to [19],

- Maintain a better driving style;

- Improve reliability of trains;

- Deliver optimum information to support decision making (operations, performance, engineering);

- Provide accurate time table information to customer.

The IRISS system provides multiple train-related services, including Service for downloading train dataset, Service for train tracking, Service for GNSS feed, Service for staff messaging, and Service for train dataset analysis.

Nottingham Scientific Limited (NSL) [20] have modified IRISS and defined the TITAN On-Board-Unit (OBU). TITAN integrates SATCOM standards like GLONASS, GPS, EGNOS, GALILEO and terrestrial standards like 3G and Wi-Fi to connect with wireless sensor networks. NSL and Avanti Communications together are providing the control station solutions to East Midland Trains (EMT). EMT has more than 43 HSR class trains operating throughout the UK.

Including the aforementioned wireless technologies, there are technologies like Fast Low Latency Access with Seamless Handoff Orthogonal Frequency Division Multiplexing (FLASH-OFDM), iBURST, Redline Communications, Faiveley, Martec, etc., which are proprietary and deployed for train access networks.

What this survey could find in reviewing these technologies is the absence of efficient and dynamic frequency allocation schemes for these networks. Without adaptive frequency allocation, wireless technologies employed together within the same heterogeneous wireless environment compete for bandwidth resources, resulting in interference and data loss. That's why recent trends suggest utilizing Cognitive Radio (CR) in railways to allocate frequency and bandwidth dynamically.

\subsection{Cognitive Radio}

After the train accident in California in 2008, the US Federal Railroad Administration (FRA) mandated the rollout of Positive Train Control (PTC). For PTC to work effectively, many different radio solutions were considered [21]. Among them is also the Rail-Cognitive Radio (Rail-CR). Amanna et al. are involved with this development [22]. In their publications, they have reasoned that network management in HSR is not trivial due to unpredictable usage, multiple users striving for bandwidth, multiple sources of interference, etc. Despite of these challenges, PTC has to maintain seamless train-to-trackside communication with locomotives, command center, and signaling. Amanna et al. therefore proposed an AI-based Software Defined Radio (SDR), which can make decisions and has the capability of adaptive learning [23]. The SDR constantly evolves and stays aware of the wireless environment through "bandwidth sniffing". SDR can allocate instantaneous free bandwidth to the system, if required. The adaptability of Rail-CR comes from persistent observation over well-defined parameters, 
i.e. carrier frequency, bit rate, modulation type, packet size, throughput, error-correction coding, etc. The learning cycle ranges from awareness, orientation to decision and action. In order to restrict the system from accessing frequencies outside the permissible range, a Policy Engine (PE) is employed and oversees the bandwidth selection according to predefined policies. The latest trend in railways communication is to move to lower frequency bands mostly to establish efficiency in control plane. Dense and over-used higher frequency spectrums have compelled SDR to use lower frequency band [23]. SDR employs two methods to optimize network management with Cognitive Engine (CE), which are, a) case-based reasoning and b) genetic algorithm.

A cognitive engine collects the physical data or parameter values in database entries, and with its optimization algorithm learns from the past decisions. Genetic Algorithms (GA), on the other hand, are a type of optimization algorithm [24] employed in machine learning. This system's CE first monitors a set of configurations for SDR under the current scenario. If the results fall beyond a particular threshold, CE tries to employ a new scenario from its historic data set. If there is no past experience suitable for the current conditions, the GA is used to develop a new strategy and enable it, which also becomes part of the historic data set.

Apart from work in the US, Europe also has a strong interest in Rail-CR in recent years. Berbineau et al. present Cognitive Radio for RaIlway through Dynamic \& Opportunistic spectrum Reuse (CORRIDOR) project in Europe [25]. The CR system in CORRIDOR carries out monitoring of maintenance data, railways data, Internet access, surveillance, and radio link. In the US most of the research is focused on deploying Rail-CR in the control plane. But the CORRIDOR project manages to also use Rail-CR in the user plane.

Berbineau et al., introduce Intelligent Mobile Terminal (IMT). IMT depends on spectrum sensing for efficient usage of free spectrum and "channel estimation \& modulation recognition", which can sense spectrum in heterogeneous networks. The entire system test bed was deployed with SNCF TGV trains in France.

However, in the US the FRA has mentioned scalability issues in CE that are not addressed in CORRIDOR. During synchronization with multiple locomotives that are trying to communicate over the same band can cause high network congestion. FRA also points out time-to-decision problems. Amanna et al. [22] have employed the Taguchi Analysis model to address this problem. According to the authors, CR with CE takes time to learn parameters in experimental test cases. Decreasing the number of required test cases is the motivation for their research. Taguchi analysis with Orthogonal Arrays (OA) can provide better configuration for GA and CE. With Taguchi analysis, 81 test cases can be optimized into 9 test cases, resulting in a low 1.5\% error in decisions. For GA to act perfectly, parameters are chosen according to crossover rate, mutation rate, population size, and maximum generation limits. But there has to be strong interaction between these parameters in order for this approach to be successful.

\section{Roadblocks for Wireless Communication in HSR}

Individual wireless standards, as pointed out in previous sections, face a variety of challenges. Most of these stem from physical conditions, such as the construction of rail cars, high temperature, voltage, shock, and vibration. It has also been observed that signals from track side equipment cannot propagate to the inside of trains due to windows and window materials. Furthermore newer railcars also shield more RF signals. Observed channel effects include fading, channel loss, fast handover etc. For fast moving transceivers, the resulting rapid fluctuations in amplitude and phase, wideband signal dispersion, inter-symbol interference, multi-carrier signal attenuation at the sub-carrier level, and "strong delayed reflection"-found particularly in satellite communication-can combine to cause signal loss. In this context, we will review the challenges HSR communication networks face.

\subsection{Channel Propagation}

HSR creates special conditions and challenges for wireless channel access, due to high velocity and rapidly changing environmental conditions. A leading cause for connectivity degradation is found in fast and slow fading effects. Another important factor is the inter-subcarrier interference [26] [27]. Other factors include "position-based" channel effects, caused by the particular impact of environment features such as viaducts, tunnels, bridges, hilly terrain and urban areas [28]-[30]. Consequently, during system simulation, fading characteristics are considered especially important in representing and evaluating wireless technologies [27] [31].

Depending upon the distance between a BS and the train, a particular loss model can be generated. Free space loss, plane earth loss, diffraction loss, multipath reception and diffraction loss model all were introduced to ad- 
dress particular effects and environments resulting from transceiver distance and obstructions in line of sight.

Especially in indoor conditions, receivers are exposed to a large number of reflected waves combining with the direct wave. Those reflected waves significantly interfere with the direct wave, thus causing fast, medium and large-scale fading. Path loss is a large-scale fading factor and can be measured to indicate BS location selection. It can be calculated by summing up each multipath component [27] ignoring antenna gains and window penetration loss. Due to large obstacles alongside the track, deep signal nulls can occur within the channel as a result of destructive combination of multiple waves. Narrowband signals in particular can be lost entirely in this fashion. Particular solutions to this problem include wide band spectrum access or using OFDM. OFDM divides the wide-band signal into multiple separate narrow-band signals. This prevents the loss of the entire signal and limits the impact of fading. Inter-symbol interference can be addressed in the same way.

Luan et al. [27] suggested a position based algorithm with Subspace Alternating Generalized ExpectationsMaximization (SAGE) to address high mobility fading due to hilly terrain. The induced fading is shadow fading that occurs due to path obstructions. This can be modeled without considering distance-related parameters. A key parameter for fading is the K-Factor, particularly for fast-moving trains. The K-Factor is defined by the ratio between dominant LOS and Rayleigh-NLOS factor components [27]. Rapid fluctuations of the K-Factor are particularly detrimental for wireless communication. The K-Factor is given as,

$$
K(\mathrm{~dB})=\log _{10} \frac{\varsigma^{2}}{\sigma^{2}}
$$

where $\varsigma$ is the LOS and $\sigma$ is the NLOS component. The K-Factor is a vital parameter in the Rician fading model.

\subsection{Handover Issues}

Handoff occurs between two cells. When a mobile user moves from one cell to another, connections need to be transferred or handed over to that cell. Due to high mobility in HSR, handover happens very frequently [31]. These handovers mostly happen where the two cells overlap. HSR with train speeds of greater than $300 \mathrm{~km} / \mathrm{h}$ and 3 - 6 km cell size can experience handoff every 20 - 30 s. With smaller cells, such as Pico cells using in RoF, this time is further reduced. During this short amount of time, multiple handover can cause packet error and packet loss.

Handover trigger probability is defined as the probability of triggering a handover when the train reaches at specific position [32]. Li et al. [32] argue that a high handover probability is required at the cell edge to maintain seamless connectivity. Adaptive handover probability [32] [33] depends on the speed of the train, and the area of the handover overlapping zone, where the best handover performance can be achieved. A premature handover can cause higher connection drop probability. It is shown that LTE-R can achieve higher than $99.8 \%$ handover probability at the cell edge, with less than 200ms handover latency (LTE) and a train speed limit of $540 \mathrm{~km} / \mathrm{h}$ [32].

Zhou et al. have argued that frequent handover [34] can degrade overall LTE-R performance. That's why some applications need to assign priority to handover calls over other calls. With location, speed and cell size information, their scheme can effectively conduct handovers in aggregate networks. Two mobile relay units can be installed to achieve better performance in non-handover zones. Call Blocking Rate (CBR) and Call Dropping Rate (CDR) are two parameters describing performance of the system. With $N_{q}$ as the number of existing calls, $R_{q}$ as the application-requested resource size; $S_{q}$ as the service time; $q$ is the traffic type index and $C$ as the capacity, the following relations can be defined according to the authors,

$$
\sum_{q} N_{q} R_{q} \leq C .
$$

We then define the handover Call Dropping Ratio as,

$$
C D R_{q}=1-\frac{N_{q}^{H}}{N_{Q}^{H}}
$$

And the Call Blocking Ratio as,

$$
C B R_{q}=1-\frac{N_{q}^{N}}{N_{Q}^{N}} .
$$


$N_{q}^{H}$ represents the total number of type-q handover calls that are admitted by the system; $N_{Q}^{H}$ denotes the total number of type-q calls that are generated. The main goal of fast handover architectures is to aim at having $\mathrm{CBR}>\mathrm{CDR}$.

There are quite a lot of handover algorithms proposed in the literature, based on adaptive and priority-based access control [35] [36]. The challenges resulting from Doppler shift and Doppler spread are addressed with solutions like Radio Environment Maps (REM) [26]. The network can be enhanced at the hardware level as well, even in NLOS areas, using better antenna systems. To get better performance in passive modulation power amplifiers and Tower Mounter Amplifiers (TMA) can be utilized [37]. For high speed of train and wind, Aluminum mount antennas provide optimum resistance. Diplexer, triplexer, fiber-bed repeaters with RF converters in tunnels and radiating cables can also improve the network performance significantly, particularly in NLOS areas. Multi-antenna schemes or Multiple Group Multiple Antenna (MGMA) are also proposed for enhanced handover [38] [39]. Table 3 provides a summary of the whole survey and suggests a trend in wireless communication.

\section{Conclusions}

The challenges in HSR wireless communications can only be adequately described and resolved when the entire wireless and geographical environment, together with the physical design of the trains, is considered jointly. There is a tight interdependence between all of these characteristics, and a variety of research efforts which have been conducted to address them for specific deployment scenarios. The driving force behind this is the desire of potential high-speed rail passengers to experience seamless Internet connectivity while onboard trains. Similarly, train control vitally depends on wireless communications, for the safety and security of its passengers and the protection of the environment. Therefore, dedicated solution for broadband wireless access for HSR is a key factor.

From this survey, it can be concluded that cognitive radios may become a standard approach to realizing these networks, for both train control operations and user accessibility, due to the advantages in dynamic spectrum management. It has also been observed in this survey that currently there are only very few approaches for train-to-train communications. Unorthodox RF strategies such as LCX onboard trains to provide an even coverage distribution along the entire passenger train have not been adequately explored in the scientific community, either.

Considering the train operators' point of view, there is no standalone wireless technology to support both user \& control communications adequately. In the past, this has led to tradeoffs in technology selection and priority-

Table 3. An overview of deployed wireless technologies in HSR.

\begin{tabular}{|c|c|c|c|c|c|c|c|c|c|c|c|}
\hline \multirow{2}{*}{ Parameters } & \multicolumn{11}{|c|}{ Wireless Technologies } \\
\hline & GSM-R & P25 & TETRA & 802.11 & WIMAX & 3G & LTE-R & RoF & LCX & Satellite & FLASH-OFDM \\
\hline $\begin{array}{l}\text { Working } \\
\text { Frequency }\end{array}$ & $\begin{array}{c}800-900 \\
\mathrm{MHz}\end{array}$ & $700 \mathrm{MHz}$ & $\sim 400 \mathrm{MHz}$ & $\begin{array}{c}2.4 / 5.8 \\
\mathrm{GHz}\end{array}$ & $\begin{array}{c}2.3 / 2.4 / 2.5 / 3.5 \\
\mathrm{GHz}\end{array}$ & $\begin{array}{c}800 / 910 \mathrm{MHz} \\
2.1 \mathrm{GHz}\end{array}$ & $\begin{array}{c}\text { Variable } \\
400-3.5 \\
\mathrm{GHz}\end{array}$ & Variable & Variable & Limited & $\begin{array}{l}450 \mathrm{MHz} \text { and } \\
\text { variable }\end{array}$ \\
\hline Data Rate & $\begin{array}{l}5-10 \\
\text { Kbps }\end{array}$ & $\begin{array}{c}40-100 \\
\text { Kbps }\end{array}$ & $\begin{array}{l}5-10 \\
\text { Kbps }\end{array}$ & $>10 \mathrm{Mbps}$ & >30 Mbps & $\begin{array}{c}>2 \text { Mbps } \\
\text { (stationary) } \\
>384 \text { Kbps } \\
\text { (Mobile) }\end{array}$ & $>10 \mathrm{Mbps}$ & $\begin{array}{l}1-10 \\
\text { Gbps }\end{array}$ & $\begin{array}{l}1-10 \\
\text { Mbps }\end{array}$ & $>2$ Mbps & $>2 \mathrm{Mbps}$ \\
\hline $\begin{array}{c}\text { IP } \\
\text { Support }\end{array}$ & Not standalone & No & No & Yes & Yes & Yes & Yes & Yes & Yes & Yes & Yes \\
\hline $\begin{array}{l}\text { Handover } \\
\text { Mechanism }\end{array}$ & $\begin{array}{l}\text { Open standard } \\
\text { (till } 500 \mathrm{~km} / \mathrm{h} \text { ) }\end{array}$ & $\begin{array}{l}\text { Open } \\
\text { standard }\end{array}$ & $\begin{array}{c}\text { Open } \\
\text { standard }\end{array}$ & Proprietary & $\begin{array}{c}\text { Open } \\
\text { standard/High } \\
\text { handover }\end{array}$ & Open standard & Open & Open; & Open & Depends & Proprietary; \\
\hline Maturity & $\begin{array}{c}\text { Mature, } \\
\text { supporteduntil } \\
2025\end{array}$ & $\begin{array}{l}\text { Mature } \\
\text { in US }\end{array}$ & Mature & Mature & $\begin{array}{l}\text { Mature, lead to } \\
\text { WIMAX } 2\end{array}$ & Mature & $\begin{array}{c}\text { Emerging } \\
\text { and offered }\end{array}$ & $\begin{array}{l}\text { Concepts like } \\
\text { "moving cell" }\end{array}$ & $\begin{array}{c}\text { Matured } \\
\text { (N700) }\end{array}$ & $\begin{array}{c}\text { Matured } \\
\text { but costly }\end{array}$ & Matured \\
\hline Market Support & Until 2025 & US & $\begin{array}{c}\text { Yes/almost } \\
\text { obsolete }\end{array}$ & Yes & Yes, decreasing & $\begin{array}{l}\text { Yes, but } \\
\text { moving to } \\
\text { LTE }\end{array}$ & $\begin{array}{l}\text { Yes, building } \\
\text { standards }\end{array}$ & Matured & $\begin{array}{l}\text { Japan, } \\
\text { Europe }\end{array}$ & $\begin{array}{l}\text { Yes, } \\
\text { in Europe } \\
\text { Thalys, } \\
\text { SNCF }\end{array}$ & Yes, Flarion \\
\hline
\end{tabular}


zation of train control communication bandwidth over passenger broadband access services. With the advent of modern technologies, this survey finds that this is changing. Increasingly, the deployment of advanced technologies in heterogeneous configurations allows concurrent train control and user communication services. LTE-R, for example, does provide a logical separation between the two planes, but lacks a physical separation between them. Without physical separation, optimum performance cannot be guaranteed. So, if only train control is the goal, GSM-R can still guarantee safe operations up to a train speed of $500 \mathrm{~km} / \mathrm{h}$. But that will not support reliable real time video surveillance or broadband access for the user plane. Wi-Fi \& Satellite can support in-train communication systems but deployment of Wi-Fi covering the entire track network in North America, for example, is infeasible. Satellite communication systems, on the other hand, cannot operate in non-line-of-sight regions and suffer from excessive latency. Heterogeneous solutions are therefore the current favored approach. In such deployments, WIMAX is preferable over Wi-Fi because it has a higher range than Wi-Fi. ITRI have successfully used WIMAX and RoF to deploy a complete communication solution in THSR. The survey could find that $4 \mathrm{G}$ networks with LTE-R increasingly could maintain train operations and user plane broadband access. Recent trends also indicate that HSR will follow the transition to 5G networks in the foreseeable future, thus alleviating remaining constraints enforced by older technologies currently in use around the globe. Further work is also needed in standardizing HSR wireless communication approaches, for interoperability across borders and across the industry.

\section{References}

[1] Fokum, D.T. and Frost, V.S. (2010) A Survey on Methods for Broadband Internet Access on Trains. IEEE Communications Surveys \& Tutorials, 12, 171-185.

[2] Yan, L. and Fang, X. (2013) Decoupled Wireless Network Architecture for High-Speed Railway. 2013 International Workshop on High Mobility Wireless Communications (HMWC), Shanghai, 1-3 November 2013, 96-100. http://dx.doi.org/10.1109/HMWC.2013.6710300

[3] Yan, L., Fang, X. and Fang, Y. (2015) Control and Data Signaling Decoupled Architecture for Railway Wireless Networks. IEEE Wireless Communications, 22, 103-111. http://dx.doi.org/10.1109/MWC.2015.7054725

[4] Shafiullah, G.M., Gyasi-Agyei, A. and Wolfs, P. (2007) Survey of Wireless Communications Applications in the Railway Industry. Proceedings of the 2nd International Conference on IEEE Wireless Broadband and Ultra Band Communication, Sydney, 27-30 August 2007, 65-70.

[5] (2005) ERTMS, SUBSET-093: GSM-R Interfaces; Class 1 Requirements.

[6] Sniady, A. and Soler, J. (2014) LTE for Railways: Impact on Performance of ETCS Railway Signaling. IEEE Vehicle Technology Magazine, 9, 69-77.

[7] Sniady, A. and Soler, J. (2013) Performance of LTE in High Speed Railway Scenarios. Proceedings of the 5th International Workshop, Nets4Cars/Nets4Trains 2013, Villeneuve d’Ascq, May 14-15, 211-222.

[8] Bertout, A. and Bernard, E. (2012) Next Generation of Railways and Metros Wireless Communication Systems. ASPECT, Institution of Railways Signal Engineers.

[9] Ghannoum, H. and Sanz, D. (2013) Internet Onboard: Technical Analysis. Proceedings of the 5th International Workshop, Nets4Cars/Nets4Trains 2013, Villeneuve d’Ascq, May 14-15, 22-30.

http://dx.doi.org/10.1007/978-3-642-37974-1 2

[10] Salaberria, I., Perallos, A., Azpilicueta, L., Falcone, F., Carballedo, R., Angulo, I., et al. (2014) Ubiquitous Connected Train Based on Train-to-Ground and Intra-Wagon Communications Capable of Providing on Trip Customized Digital Services for Passengers. Sensors, 14, 8003-8025. http://dx.doi.org/10.3390/s140508003

[11] Shrestha, P., Hempel, M., Sharif, H. and Mehrvarzi, S. (2013) A Comparative Analysis of Mobile WiMAX 2.5 GHz and $3.65 \mathrm{GHz}$ for Freight Railroad Applications. 9th International Conference on Innovations in Information Technology (IIT), Abu Dhabi, 17-19 March 2013, 186-191.

[12] Lannoo, B., Colle, D., Pickavet, M. and Demeester, P. (2007) Radio-over-Fiber-Based Solution to Provide Broadband Internet Access to Train Passengers (Topics in Optical Communications). IEEE Communications Magazine, 45, 56-62. http://dx.doi.org/10.1109/MCOM.2007.313395

[13] Pleros, N., Vyrsokinos, K., Tsagkaris, K. and Tselikas, N. (2009) A 60 GHz Radio-over-Fiber Network Architecture for Seamless Communication with High Mobility. Journal of Lightwave Technology, 27, 1957-1967. http://dx.doi.org/10.1109/JLT.2009.2022505

[14] De Greve, F., Lannoo, B., Peters, L., Van Leeuwen, T., Van Quickenborne, F., Colle, D., et al. (2005) FAMOUS: A Network Architecture for Delivering Multimedia Services to Fast Moving Users. Wireless Personal Communication, 


\section{3, 281-304. http://dx.doi.org/10.1007/s11277-005-0573-2}

[15] Han, T. and Ansari, N. (2015) RADIATE: Radio over Fiber as an Antenna Extender for High-Speed Train Communications. IEEE Wireless Communications, 22, 130-137.

[16] Terada, M. and Teraoka, F. (2012) Providing a High-Speed Train with a Broadband and Fault Tolerant IPv4/6 NEMO Environment. IEEE Globecom Workshops, Anaheim, 3-7 December 2012, 1052-1056. http://dx.doi.org/10.1109/GLOCOMW.2012.6477723

[17] Guan, J. and Zhou, K. (2013) Analysis of the Radiation Field Distribution Characteristics of the LTE-R Leakage Coaxial Cable in the High-Speed Train. 5th IEEE International Symposium on Microwave, Antenna, Propagation and EMC Technologies for Wireless Communications, Chengdu, 29-31 October 2013, 728-731. http://dx.doi.org/10.1109/MAPE.2013.6689857

[18] Sanz, D. (2006) Satellite Technolgies for Broadband Internet Access Onboard High Speed Train. SNCF-Direction de l'Innovation et de la Recherche, Paris, France.

[19] European Space Agency (ESA) ARTES Applications, Integrated Railways via Integrated Satellite Systems (IRISS). https://artes-apps.esa.int/projects/iriss

[20] Kruijff, M., Dumville, M., Hutchinson, M. and Ginati, A. (2011) Intelligent Railways via Integrated Satellite Services (IRISS). IAC-11-B5.2.7.

[21] Federal Railroad Administration, U.S. Department of Transportation (2013) Railway Cognitive Radio to Enhance Safety, Security, and Performance of Positive Train Control. DOT/FRA/ORD-13/09.

[22] Amanna, A., Ali, D., Gadhiok, M., Price, M. and Reed, J. (2012) Cognitive Radio Engine Parametric Optimization Utilizing Taguchi Analysis. EURASIP Journal on Wireless Communications and Networking, 2012, 5. http://dx.doi.org/10.1186/1687-1499-2012-5

[23] Amanna, A., Gadhiok, M., Price, M., Reed, J., Siriwongpairat, W. and Himsoon, T. (2010) Railway Cognitive Radio. IEEE Vehicular Technology Magazine, 5, 82-89. http://dx.doi.org/10.1109/MVT.2010.938275

[24] U.S. Department of Transportation, Federal Rail Road Administration. Rail-CR: Railroad Cognitive Radio, Research Results. FRA Office of Railroad Policy \& Development.

[25] Bebineau, M., Masson, E., Cocheril, Y., Kalakech, A., Ghys, J.P., Dayoub, I., et al. (2014) Cognitive Radio for High Speed Railway through Dynamic and Opportunistic Spectrum Reuse. Transport Research Arena 2014, Paris, 14-17 April 2014, 1-10.

[26] Li, J. and Zhao, Y. (2012) Radio Environment Map-Based Cognitive Doppler Spread Compensation Algorithms for High-Speed Rail Broadband Mobile Communications. EURASIP Journal on Wireless Communication and Networking, 2012, 263. http://dx.doi.org/10.1186/1687-1499-2012-263

[27] Luan, F.Y., Zhang, Y., Xiao, L.M., Zhou, C.H. and Zhou, S.D. (2013) Fading Characteristics of Wireless Channel on High-Speed Railway in Hilly Terrain Scenario. International Journal of Antennas and Propagation, 2013, Article ID: 378407.

[28] Guan, K., Zhong, Z. and Ai, B. (2011) Assessment of LTE-R Using High Speed Railway Channel Model. 3rd International Conference on Communications and Mobile Computing, Qingdao, 18-20 April 2011, 461-464. http://dx.doi.org/10.1109/cmc.2011.34

[29] Zhou, T., Tao, C., Liu, L., Qiu, J. and Sun, R. (2012) High-Speed Railway Channel Measurements and Characterizations: A Review. Journal of Modern Transportation, 20, 199-205. http://dx.doi.org/10.1007/BF03325799

[30] Ai, B., Cheng, X., Kurner, T., Zhong, Z., Guan, K., He, R., et al. (2014) Challenges toward Wireless Communications for High-Speed Railway. IEEE Transactions on Intelligent Transportation System, 15, 2143-2158. http://dx.doi.org/10.1109/TITS.2014.2310771

[31] Tseng, M.-C. and Cheng, M.-H. (2012) Experimental Study of Fading Characteristics for Wireless Communications in High-Speed Railway Environments. Proceedings for IEEE Antennas and Propagation Society International Symposium (APSURSI), Chicago, 8-14 July 2012, 1-2.

[32] Li, J., Tian, L., Zhou, Y. and Shi, J. (2012) An Adaptive Handover Trigger Scheme for Wireless Communications on High Speed Rail. IEEE International Conference on Communications (ICC), Ottawa, 10-15 June 2012, 5185-5189. http://dx.doi.org/10.1109/icc.2012.6364484

[33] Xu, Q., Ji, H., Li, X. and Zhang, H. (2015) Admission Control Scheme for Service Dropping Performance Improvement in High-Speed Railway Communication Systems. IEEE Transactions on Vehicular Technology, PP, 1. http://dx.doi.org/10.1109/tvt.2015.2458512

[34] Zhou, Y. and Ai, B. (2014) A Position-Based Access Scheme for High-Speed Railway Communications. IEEE International Conference on Signal Processing, Communications and Computing (ICSPCC), Guilin, 5-8 August 2014, 608612. http://dx.doi.org/10.1109/ICSPCC.2014.6986265 
[35] Zhao, Y., Li, X. and Ji, H. (2012) Radio Admission Control Scheme for High-Speed Railway Communication with MIMO Antennas. IEEE International Conference on Communications (ICC), Ottawa, 10-15 June 2012, 5005-5009. http://dx.doi.org/10.1109/icc.2012.6363947

[36] Kwan, R., Arnott, R. and Kubota, M. (2010) On Radio Admission Control for LTE Systems. IEEE 72nd Vehicular Technology Conference Fall, Ottawa, 6-9 September 2010, 1-5. http://dx.doi.org/10.1109/VETECF.2010.5594566

[37] Radio Frequency Systems (2009) Solutions for High-Speed Rail and Expressway Wireless Coverage in China. August.

[38] Tian, L., Li, J., Huang, Y., Shi, J. and Zhou, J. (2012) Seamless Dual-Link Handover Scheme in Broadband Wireless Communication Systems for High-Speed Rail. IEEE Journals on Selected Areas of Communication, 30, 708-718. http://dx.doi.org/10.1109/JSAC.2012.120505

[39] Luo, W.T., Fang, X.M., Cheng, M. and Zhao, Y.J. (2013) Efficient Multiple-Group Multiple-Antenna (MGMA) Scheme for High-Speed Railway Viaducts. IEEE Transactions on Vehicular Technology, 62, 2558-2569. http://dx.doi.org/10.1109/TVT.2013.2244106 